\title{
Transcriptional Pathways Associated with Skeletal Muscle Changes after Spinal Cord Injury and Treadmill Locomotor Training
}

\author{
Celine Baligand, ${ }^{1}$ Yi-Wen Chen, ${ }^{2,3}$ Fan Ye, ${ }^{4}$ Sachchida Nand Pandey, ${ }^{2}$ \\ San-Huei Lai, ${ }^{2}$ Min Liu, ${ }^{4}$ and Krista Vandenborne ${ }^{4}$ \\ ${ }^{1}$ Department of Physiology and Functional Genomics, University of Florida, Gainesville, FL 32610, USA \\ ${ }^{2}$ Center for Genetic Medicine Research, Children's National Health System, Northwest, Washington, DC 20010, USA \\ ${ }^{3}$ Department of Integrative Systems Biology, George Washington University, Northwest, Washington, DC 20010, USA \\ ${ }^{4}$ Department of Physical Therapy, University of Florida, Gainesville, FL 32610, USA
}

Correspondence should be addressed to Krista Vandenborne; kvandenb@phhp.ufl.edu

Received 11 February 2015; Accepted 19 May 2015

Academic Editor: Christian Guelly

Copyright @ 2015 Celine Baligand et al. This is an open access article distributed under the Creative Commons Attribution License, which permits unrestricted use, distribution, and reproduction in any medium, provided the original work is properly cited.

\begin{abstract}
The genetic and molecular events associated with changes in muscle mass and function after SCI and after the implementation of candidate therapeutic approaches are still not completely known. The overall objective of this study was to identify key molecular pathways activated with muscle remodeling after SCI and locomotor training. We implemented treadmill training in a wellcharacterized rat model of moderate SCI and performed genome wide expression profiling on soleus muscles at multiple time points: 3, 8, and 14 days after SCI. We found that the activity of the protein ubiquitination and mitochondrial function related pathways was altered with SCI and corrected with treadmill training. The BMP pathway was differentially activated with early treadmill training as shown by Ingenuity Pathway Analysis. The expression of several muscle mass regulators was modulated by treadmill training, including Fst, Jun, Bmpr2, Actr2b, and Smad3. In addition, key players in fatty acids metabolism ( $L p l$ and Fabp3) responded to both SCI induced inactivity and reloading with training. The decrease in Smad3 and Fst early after the initiation of treadmill training was confirmed by RT-PCR. Our data suggest that TGF $\beta / S$ mad 3 signaling may be mainly involved in the decrease in muscle mass observed with SCI, while the BMP pathway was activated with treadmill training.
\end{abstract}

\section{Introduction}

Spinal cord injury (SCI) is one of the most disabling health problems faced by adults today. One of the physiological changes secondary to SCI is progressive muscle atrophy. The loss of muscle mass after spinal cord injury has been well documented, with patients with complete SCI having about 20-55\% muscle atrophy [1] and patients with incomplete SCI showing about $20-30 \%$ atrophy [2] within 6 months to 1 year after SCI. Muscle wasting does not only impact the care and lifestyle of patients after SCI but also has significant health implications, increasing the patients' risk to develop secondary complications such as bone demineralization, diabetes, and cardiovascular disease [3, 4]. Maintenance of muscle mass is essential to metabolic health and is necessary to maximize functional gain from rehabilitation strategies after SCI.

A potential rehabilitation intervention in the treatment of individuals with SCI is the use of repetitive locomotor training to promote neural plasticity. This approach was derived from animal and human studies showing that stepping can be generated by virtue of the neuromuscular system's responsiveness to phasic peripheral sensory information associated with locomotion, in the presence of central pattern generator (reviewed in [5]). Our group and others have shown that locomotor training can induce substantial 
recovery in muscle size and muscle function in transected [6] and moderate contusion injury animal models of SCI [7-9]. Some studies suggest that this may reflect enhanced synthesis of growth factors within the central nervous system [10], but other potential activity-dependent molecular changes remain unknown, in particular the ones occurring locally in the muscle in parallel with atrophy and hypertrophy.

Muscle atrophy depends on the balance between protein breakdown, protein synthesis rates, and apoptosis. It has been attributed to the activation of various protein degradation pathways in several models of disuse, such as denervation, unloading, cachexia, or aging [11, 12]. Previous work in animal models showed that alterations in the protein ubiquitination and energy production related pathways are common features of the atrophy process [1316]. The activation of growth factors such as insulin-like growth factors (IGFs), myogenic regulatory factors (MRFs) [17], transforming growth factors (TGFs) [18], and the bone morphogenic proteins (BMPs) [19] has also been shown to play an important role in muscle atrophy and hypertrophy. However, the activation or predominance of the different pathways involved can be specific to the condition inducing atrophy [20]. To our knowledge, only one study used gene profiling in human muscle samples to perform a general screening of the pathways activated in skeletal muscle after SCI [21]. However, the specific molecular signaling changes that occur after SCI and subsequent recovery and/or rehabilitation intervention remain largely unknown.

A better understanding of the molecular events regulating protein synthesis and degradation after SCI and locomotor training and their temporal relationship to changes in muscle mass is of considerable clinical importance and has farreaching implications for posttraumatic health care. Therefore, the overall objective of this study was to identify key molecular pathways activated with muscle remodeling after SCI and during locomotor training. We implemented treadmill training in a well-characterized rat model of moderate SCI [9, 22-24] and performed genome wide expression profiling with microarray on soleus muscles at multiple time points during the course of SCI (prior and 3, 8, and 14 days after SCI) and during the course of a treadmill training intervention initiated 7 days after injury ( 8 and 14 days after SCI). We used two different approaches for data analyses. First, we took an unsupervised approach and identified molecular pathways affected most at each time point. We then targeted genes that are known to be involved in muscle remodeling and may play important roles in the process.

\section{Material and Methods}

2.1. Study Design. In this cross-sectional study, six groups of rats were studied, including a control group, three SCI groups at three time points (days 3, 8, and 14), and 2 SCI groups with treadmill training at two time points (days 8 and 14). In the latter groups, the treadmill was initiated 7 days after SCI. For the analysis of changes during the course of SCI, comparisons were done with reference to control samples for each time point after SCI (days 3, 8, and 14). For the analysis of the effects of treadmill training on SCI, samples from the treadmill trained SCI rats were compared to samples collected from SCI rats with no training. Differences between the trained muscles and untrained muscles at a specific time point (day 8 or day 14) were determined.

2.2. Animals. Thirty-six adult female Sprague Dawley rats (16 weeks of age, 260-280 $\mathrm{g}$ at the beginning of the study) were obtained from Charles River Laboratories and housed in a temperature $\left(22 \pm 1^{\circ} \mathrm{C}\right)$ and humidity $(50 \pm 10 \%)$ controlled room with 12:12 hours light:dark cycle. Animals were provided rodent chow and water ad libitum and were given 1 week to acclimatize to the environment. Animals were sacrificed at one of the following time points and conditions: 3 days after SCI (SCI3d, $n=6$ ), 8 days after SCI (SCI8d, $n=6$ ), 14 days after SCI (SCI14d, $n=6$ ), 8 days after SCI and after 3 treadmill training sessions (SCI8d + TM, $n=6$ ), and 14 days after SCI and after 5 days of repeated treadmill training sessions (SCI14d + TM). Age-matched control animals (CTR, $n=6$ ) were used as the baseline. Experimental animals were given access to transgenic dough diet (Bio-Serv, NJ, product number S3472), placed on the bottom of the cage, to ensure adequate food intake. All procedures were performed in accordance with the US Government Principle for the Utilization and Care of Vertebrate Animals and were approved by the Institutional Animal Care and Use Committee at the University of Florida.

2.3. Spinal Cord Injury Procedure. All surgical procedures were performed under aseptic conditions. Moderate contusion SCI was produced using a NYU-MASCIS injury device as previously described $[25,26]$. Briefly, the animals were deeply anesthetized with a combination of ketamine (90 mg/kg body weight) and xylazine ( $8 \mathrm{mg} / \mathrm{kg}$ body weight) and a dorsal laminectomy was performed at the thoracic vertebral level T7-T9 to expose the spinal cord [27]. Clamps attached to the spinous processes of $\mathrm{T} 7$ and $\mathrm{T} 9$ stabilized the vertebral column. Contusion was produced by dropping a $10 \mathrm{~g}$ cylinder from a height of $25 \mathrm{~mm}$ onto the T8 segment of the spinal cord. Analgesia was given in the form of buprinex $(0.025 \mathrm{mg} / \mathrm{kg})$ and ketoprofen $(22 \mathrm{mg} / \mathrm{kg})$ once daily over the first 36 hrs after SCI. The animals were housed individually and kept under vigilant postoperative care, including daily examination for signs of distress, weight loss, dehydration, and bladder dysfunction. Manual expression of bladders was performed 2-3 times daily, as required, and animals were monitored for the possibility of urinary tract infection.

2.4. Locomotor Training. Quadrupedal locomotor training was initiated on postoperative day 7. Training consisted of $20 \mathrm{~min}$ stepping sessions on a treadmill. Training was performed 3 times in the SCI8d + TM group (2 times on day 7 and one time on day 8) and was repeated twice a day for 5 days in the SCI14d - TM group. When necessary, body weight support was manually provided by the trainer. The level of body weight support was adjusted to make sure that the hindlimbs of the animals did not collapse and was gradually removed as locomotor capability improved. During 
the first day of training, assistance was provided to place the rat hindpaws in plantar-stepping position during training. Typically, rats started stepping when they experienced some load on their hindlimbs.

2.5. Tissue Collection. Left soleus muscles were harvested in all groups. In the SCI8d + TM and SCI14d + TM groups, muscle samples were harvested 8 hours after the end of the last treadmill training session. Briefly, rats were anesthetized with isoflurane (3\% for induction, $1-2 \%$ for maintenance), and a small dorsal, midline incision was made to expose the gastrocnemius-soleus complex. The soleus was carefully separated from the gastrocnemius, harvested, and weighed. The sample was rapidly frozen in isopentane, precooled in liquid nitrogen, and subsequently stored at $-80^{\circ} \mathrm{C}$.

$t$-test was used to determine the statistical significance $(p<0.05)$ of the changes in muscle mass.

2.6. Expression Profiling. GeneChip Rat Genome 2302.0 Array microarrays containing approximately 30,000 transcripts were used for the expression profiling experiment. Standard procedures including total RNA isolation, cDNA synthesis, cRNA labeling, microarray hybridization, and image acquisition were done as described in the manufacturer's protocol and our previous publications [20, 28]. Briefly, total RNA was isolated with TRIzol reagent (Invitrogen) and then purified with RNeasy MinElute Cleanup Kit (Qiagen). Two hundred nanograms of total RNA from each sample was reverse-transcribed to double-stranded cDNA followed by in vitro cRNA synthesis using one-cycle target labeling and control reagents and protocol (Affymetrix). Biotin-labeled cRNA was then purified using GeneChip Sample Cleanup Module (Affymetrix) and fragmented randomly prior to hybridizing to the microarrays overnight. Each array was washed and stained using the Affymetrix Fluidics Station 450 and then scanned using the GeneChip Scanner 3000. The quality control criteria developed at Children's National Medical Center Microarray Center for each array were followed [6].

Generation of hybridization signals of the microarrays was done using Microarray Suite 5.0 (MAS 5.0) (Affymetrix, CA) as previously described [21, 29-32]. After the absolute analysis, the gene expression values were imported into GeneSpring 11.0 (Silicon Genetics) for data filtering and statistical analysis. First, genes were filtered with numbers of present calls across the 36 arrays analyzed. Genes with at least 4 present calls (detected by more than $10 \%$ of the arrays) were selected for statistical analysis. We identified 31099 probe sets that met this filtering criterion. In GeneSpring, $t$-test was performed and probe sets showing significant $(p<0.05)$ expression changes were retained for pathway analysis. No additional fold change filters were used. The comparisons were done by comparing samples of each time point after SCI (days 3, 8, and 14) to the control time point (day 0), respectively. The treadmill trained samples were compared to the SCI samples collected at the same time point to obtain differences between the trained muscles and untrained muscles at a specific time point (day 8 and day 14).
TABLE 1: Primer sequences used for quantitative RT-PCR.

\begin{tabular}{lll}
\hline Genes & Primer & Primer sequence \\
\hline \multirow{2}{*}{ Gapdh } & Forward & F-5'-TCCGCCCCTTCCGCTGATG-3' \\
& Reverse & R-5'-CACGGAAGGCCATGCCAGTGA-3' \\
\hline \multirow{2}{*}{ Smad3 } & $\begin{array}{l}\text { Forward } \\
\text { Reverse }\end{array}$ & $\begin{array}{l}\text { F-5'-AAGATACCCCCAGGCTGC-3' } \\
\text { R-5'-CTGTCTGTCTCCTGTACTC-3' }\end{array}$ \\
\hline \multirow{2}{*}{ Myf6 } & Forward & F-5'-CTAAGGAAGGAGGAGCAAG-3' \\
& Reverse & R-5' -TGTTCCAAATGCTGACTGAG-3' \\
\hline \multirow{2}{*}{ Fst } & Forward & F-5'-GTGTATCAAAGCAAAGTCTTG-3' \\
& Reverse & R-5'-GCTCATCGCAGAGAGCA-3' \\
\hline
\end{tabular}

To investigate molecular networks and pathways associated with gene lists in this study, Ingenuity Pathway Analysis (IPA) (Ingenuity Systems) was used with default settings to identify gene interactions and to prioritize molecular pathways differentially affected in different groups. Hierarchical clustering was performed using GeneSpring software to visualize transcripts showing coordinate regulation as a function of time.

The significance of the association between the genes in each dataset and the canonical pathway was determined by Fisher's exact test. The $p$ values were calculated to determine the probability of the association between the genes. All profiles have been made publicly accessible via NCBI GEO (number GSE45550) (http://www.ncbi.nlm.nih.gov/geo/).

2.7. Reverse Transcription and Quantitative RT-PCR Analysis. Reverse transcription and quantitative RT-PCR (qRT-PCR) were performed as previously described [17]. Briefly, total RNA $(2 \mu \mathrm{g})$ was reverse-transcribed to cDNA using oligo(dT) primer $(0.5 \mu \mathrm{g} / \mu \mathrm{L})$ and reagents from Invitrogen. cDNA was amplified in triplicate in SYBR Green PCR Master Mix (Applied Biosystems). The thermal cycling conditions included $95^{\circ} \mathrm{C}$ for $10 \mathrm{~min}$, followed by 40 cycles of amplification at $95^{\circ} \mathrm{C}$ for $15 \mathrm{~s}$ and $60^{\circ} \mathrm{C}$ for $1 \mathrm{~min}$. Primer sequences used for rat myogenic factor 6 (MYF6 6/MRF4), follistatin (FST), mothers against decapentaplegic homolog 3 or SMAD family member 3 (SMAD3), and glyceraldehyde 3-phosphate dehydrogenase (GAPDH), which served as an internal control, are provided in Table 1 . All primers were tested for nonspecific amplicons and primer dimers by visualizing PCR products on $2 \%$ agarose gels before performing qRT-PCR. The $\Delta \Delta \mathrm{CT}$ value method (where CT is cycle threshold) was used to determine fold differences as described previously [17].

\section{Results}

3.1. Changes in Soleus Muscle Wet Weight after SCI and Treadmill Training. Soleus muscle wet weights are presented in Figure 1. SCI resulted in a rapid loss in muscle weight 3 and 8 days after injury $(-25 \%, p=0.0005)$. By day 14 , muscle weight was still lower than in controls without reaching significance $(-16 \%, p=0.056)$. However, 5 days of training significantly increased muscle wet weight (SCI14d + TM, $p=0.005$ ). 
TABLE 2: Five most significantly activated pathways for each comparison of the study.

\begin{tabular}{|c|c|c|c|}
\hline Group/comparison & 3 days & 8 days & 14 days \\
\hline \multirow{5}{*}{$\begin{array}{l}\text { No treadmill training } \\
\text { versus control }\end{array}$} & Protein ubiquitination pathway & Mitochondrial dysfunction & Mitochondrial dysfunction \\
\hline & Mitochondrial dysfunction & $\begin{array}{l}\text { Valine, leucine, and isoleucine } \\
\text { degradation }\end{array}$ & $\begin{array}{l}\text { Synthesis and degradation of } \\
\text { ketone bodies }\end{array}$ \\
\hline & Oxidative phosphorylation & Propanoate metabolism & Oxidative phosphorylation \\
\hline & $\begin{array}{l}\text { Regulation of eIF4 and p70S6K } \\
\text { signaling }\end{array}$ & Butanoate metabolism & Citrate cycle \\
\hline & Ubiquinone biosynthesis & Pyruvate metabolism & Ephrin receptor signaling \\
\hline \multirow{5}{*}{$\begin{array}{l}\text { Treadmil training versus } \\
\text { no training }\end{array}$} & & Protein ubiquitination pathway & Estrogen receptor signaling \\
\hline & & Amyloid processing & EIF2 signaling \\
\hline & & BMP signaling pathway & $\begin{array}{l}\text { Regulation of eIF4 and } \\
\text { p70S6K signaling }\end{array}$ \\
\hline & & $\begin{array}{l}\text { Role of BRCA1 in DNA damage } \\
\text { response }\end{array}$ & Glutamate metabolism \\
\hline & & Hereditary breast cancer signaling & Granzyme B signaling \\
\hline
\end{tabular}

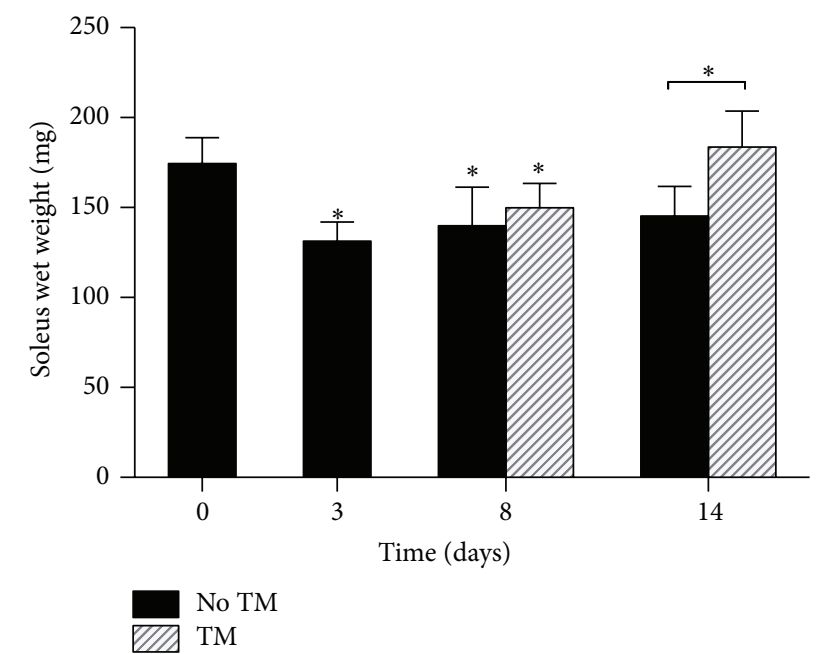

FIGURE 1: Muscle wet weight was significantly decreased on days 3 and 8 as compared to day 0 and significantly increased in the treadmill training group as compared to untrained animals on day 14. ${ }^{*} p<0.05$.

\subsection{The Highest Number of Differentially Expressed Transcripts} Was Observed 3 Days after SCI. The largest number of genes differentially expressed compared to controls was found 3 days after SCI (Figure 2). In the treadmill trained groups, the largest changes were found on day 14, compared to untrained animals (Figure 2). In the untrained group, about $60 \%$ of the genes were downregulated at each time point, which was reversed in the treadmill training group on day 8 with $63 \%$ of the genes upregulated. To identify the major molecular pathways affected in each condition, Ingenuity Pathway Analysis (IPA) was performed. Table 2 shows the top 5 canonical pathways for each comparison. Genes Were Altered following SCI. At the early time point

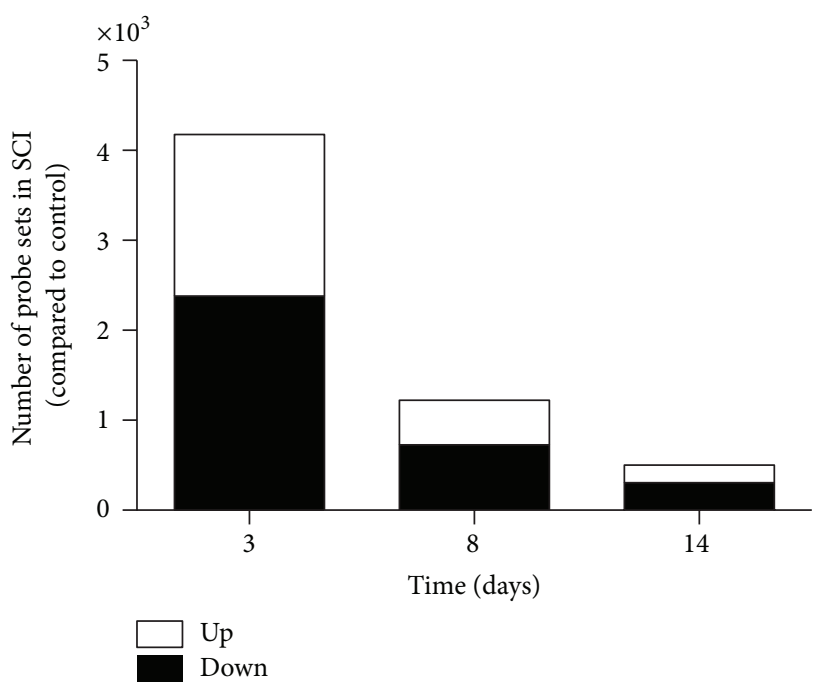

Figure 2: Number of probe sets differentially expressed in control and SCI rat soleus muscles decreased 8 and 14 days after SCI.

(day 3), we found that the protein ubiquitination pathway was largely activated. Indeed, $90 \%$ of the genes identified by IPA showed increase in gene expression. In particular, the expression of many proteasome subunits (PSMs) and ubiquitin specific peptidases and enzymes was increased (Suppl. Table 2 in Supplementary Material available online at http://dx.doi.org/10.1155/2015/387090). However, changes in this pathway were no longer significant on days 8 and 14 as compared to control levels. On the other hand, the mitochondrial dysfunction and oxidative phosphorylation pathways were consistently ranked among the top 5 most significant pathways at all time points of the study (days 3, 8 , and 14) (Table 2). More than 95\% of the genes identified in these two pathways were downregulated 3 days after SCI (Suppl. Tables 2, 3, and 4). In particular, the expression of the NADH dehydrogenase subunits (NDUF) genes, the succinate 
dehydrogenase complex subunits (SDH) genes, and the ATP synthase genes showed a decrease in gene expression.

3.4. Treadmill Training Rapidly Reversed the Changes in Protein Ubiquitination, Translation Factors, and Mitochondrial Function Pathway. The protein ubiquitination pathway was rapidly affected by treadmill training. While the majority of the PSMs were upregulated on day 3, an overall significant decrease was observed after 3 sessions of treadmill training. In the Eif 2 and the eif4/p70sk6 pathways, the expression of the eukaryotic translation initiation factors (EIFs) subunits and kinases underwent overall negative fold changes on day 3 (Suppl. Table 2), which was corrected with training (Suppl. Table 4). In addition, the expression of NDUFs ATP synthases, $\operatorname{Cox} 8 A$ and $\operatorname{Cox} 7 A 2 L$, was upregulated in trained animals on day 14 , also showing a corrective effect of TM on muscle oxidative metabolism.

3.5. TGF- $\beta /$ Smad and BMP Signaling Pathways Are Involved in the Muscle Remodeling Process during the Course of SCI and Early Treadmill Training. Because the bone morphogenic proteins (BMPs) signaling pathway was significantly altered on day 8 in the trained group (ranked number $3, p<0.005$ ), we examined the changes in gene expression of some key players in this pathway and in the TGF $\beta$ pathway. To explore possible downstream changes, we also looked into the gene expression of the different smads associated with these pathways (Smad1, Smad3, Smad4, Smad5, Smad6, and Smad8), in particular on day 3 , and with acute training on day 8 .

On day 3 in the BMP pathway, Smad4 $(+1.8, p<0.05)$, Smad1 $(+1.8, p<0.05)$, and Smad5 $(+1.3, p<0.05)$ gene expression was upregulated. Smad3 did not show any significant changes on day 3 , while the activin receptor $2 \mathrm{~B}($ Acvr $2 B)$ was downregulated $(-1.6, p<0.05)$. In parallel, Smad6, inhibitor of Bmpr2 activation, was decreased $(-2.3, p<0.05)$.

In SCI8d, Smad1 gene expression was increased $(+1.6$ fold, $p<0.05)$. In addition, both follistatin (Fst) and Smad3 expression were increased $(+2.5$ fold, $p<0.05,+2.0$ fold, $p<0.05$, resp.) as compared to controls (Figure 3 ).

In SCI + TM on day 8, Fst was significantly decreased $(-1.5$ fold, $p<0.05)$. Smad 3 was also found to be significantly downregulated $(-1.3$ fold, $p<0.05)$. The expression of the BMP complex 3 (Bmp3) was decreased $(-2.35, p<0.05)$. Importantly, Bmpr 2 was concomitantly overexpressed with treadmill training as compared to SCI only (+1.6 fold, $p<$ $0.05)$. As part of the genes reported in the BMP pathway activated with training on day 8 , Jun followed the same large and transient increase $(+1.5, p<0.05)$ as seen in Bmpr2 (Figure 3).

3.6. Genes Involved in Myogenesis and Muscle Regeneration Were Affected by Treadmill Training. In addition to genes identified by IPA, we specifically studied genes involved in myogenesis, lipid metabolism, and fiber type switches, with a particular focus on those that were affected by 5-day treadmill training.

Igfbp5, modulator of IGF1 function in skeletal muscle, showed a significant decrease 3 days after SCI, followed by a large increase on day 14 . This upregulation was not observed in the treadmill training group.

Three days after injury, dramatic changes were observed in myogenic regulatory factors (MRFs), including $M y f 6$ and myogenin (Myog) (Figure 4). The Myog expression pattern was not affected by treadmill training. On the other hand, treadmill training induced an additional increase $(p<0.05)$ in $M y f 6$ after the first 3 sessions as compared to untrained animals.

3.7. Fatty Acid Metabolism and Fiber Type Switch Related Genes Showed High Sensitivity to SCI and Treadmill Training. Lipoprotein lipase $(\mathrm{LPl})$ and Fabp3 gene expression were dramatically decreased 3 days after SCI as compared to controls and maintained low expression levels on days 8 and 14 after SCI. While the first sessions of locomotor training did not significantly affect $L p l$ expression, it was completely restored on day $14(+2.32$ fold, $p<0.005)$ as shown in Figure 5.

As early as 3 days after SCI and throughout the experiment, several fast-twitch fiber markers genes (Mhyl, Mybph, and Myh4) showed large and significant changes (Figure 6). In parallel, the expression of several slow-twitch and vasculature smooth muscles markers (Myh3 and Myl2) was decreased $(p<0.04)$ (Figure 6). Treadmill training had a significant reverse effect on the expression of some fiber type related genes, such as the fast-twitch markers Myh1 and Myh4.

3.8. $R T-q P C R$ Validation. To validate microarray findings, we selected 3 genes (Fst, Smad3, and Myf6) that were found to be significantly affected by SCI and showed reversed changes after the initial treadmill training. RT-qPCR confirmed that Fst mRNA levels were significantly higher on day 8 after SCI compared to controls ( +2.5 fold, $p<0.05)$ and decreased in the trained group compared to untrained (Figure 7). Smad3 expression was also significantly decreased with acute treadmill training $(-1.5$ fold, $p<0.05)$. On the other hand, the increase in Myf6 in SCI on day 8 was not confirmed (Figure 7(c)).

\section{Discussion}

The genetic and molecular events associated with changes in muscle mass and function after SCI and after the implementation of candidate therapeutic approaches are still not completely known. We used a well-characterized rat model of moderate SCI combined with treadmill training as a rehabilitation strategy to explore the pathways and genes involved in these conditions. The unique design of our study and the use of genome wide analysis allowed the identification of several major canonical pathways involved in protein synthesis and muscle metabolism regulation after SCI and treadmill training. In particular, the activity of the protein ubiquitination and mitochondrial function related pathways was altered with SCI and corrected with treadmill training. Of particular interest, the BMP pathway was differentially activated with early treadmill training as shown by IPA. The expression of several muscle mass regulators was modulated 


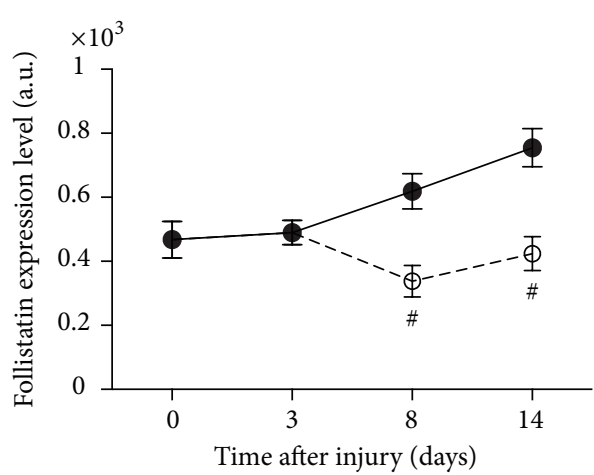

(a)

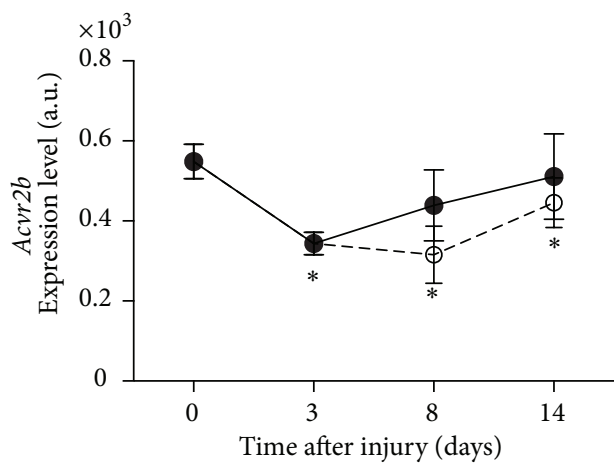

(c)

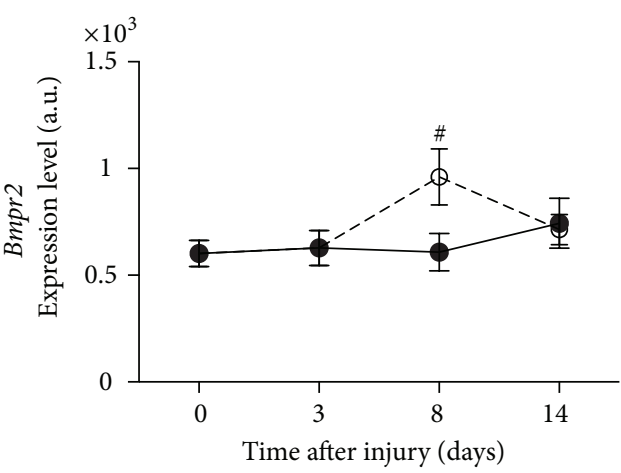

(e)

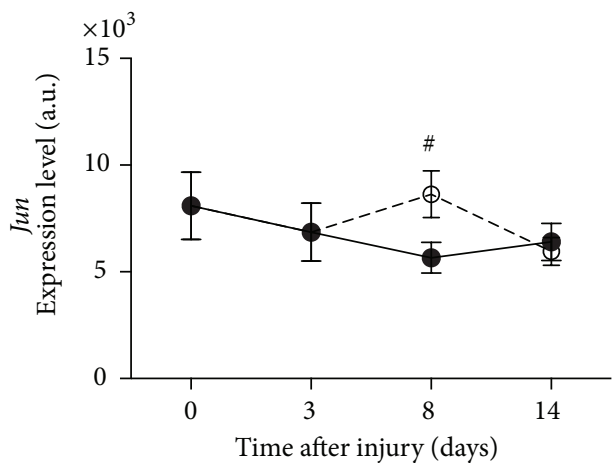

- No TM

○ TM

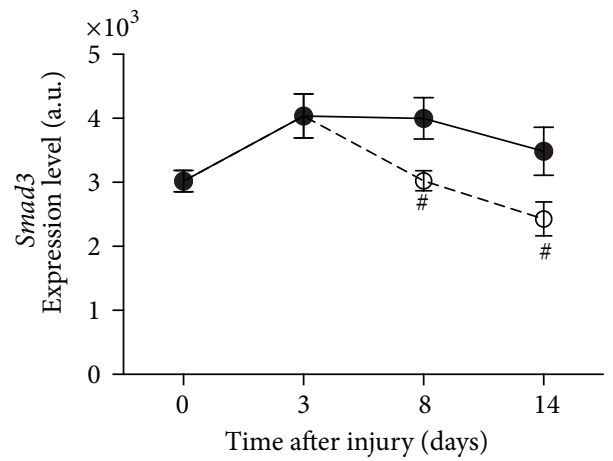

(b)

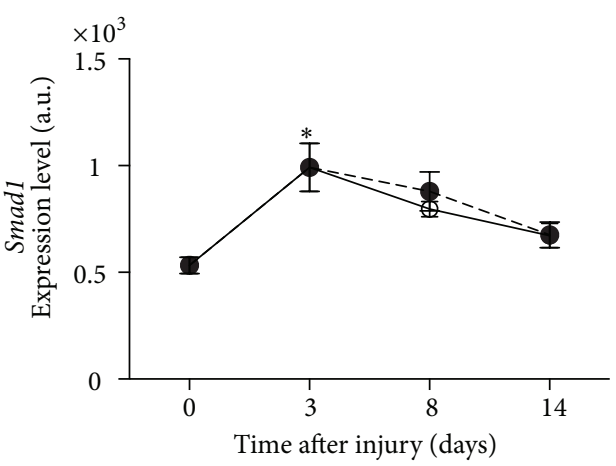

(d)

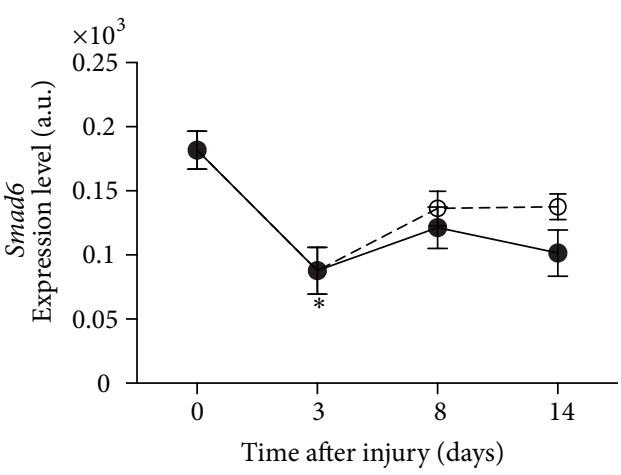

(f)

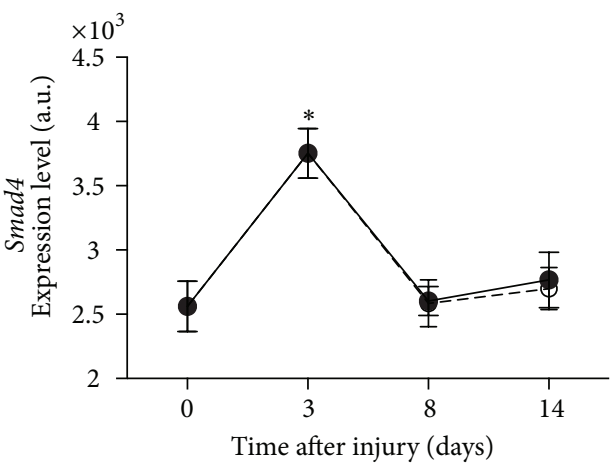

- No TM

O TM

(g)

(h)

Figure 3: BMP/TGF $\beta$ pathway related genes. Gene expression level changes in Fst (a), Smad3 (b), Acvr2b (c), Smad1 (d), Bmpr2 (e), Smad6 (f), Jun (g), and Smad4 (h) in soleus of trained and untrained SCI animals. All expression levels are referenced to a control sample's GAPDH levels. ${ }^{*}$ Significantly different from controls $(p<0.05)$. " Significantly different from untrained animals $(p<0.05)$. 


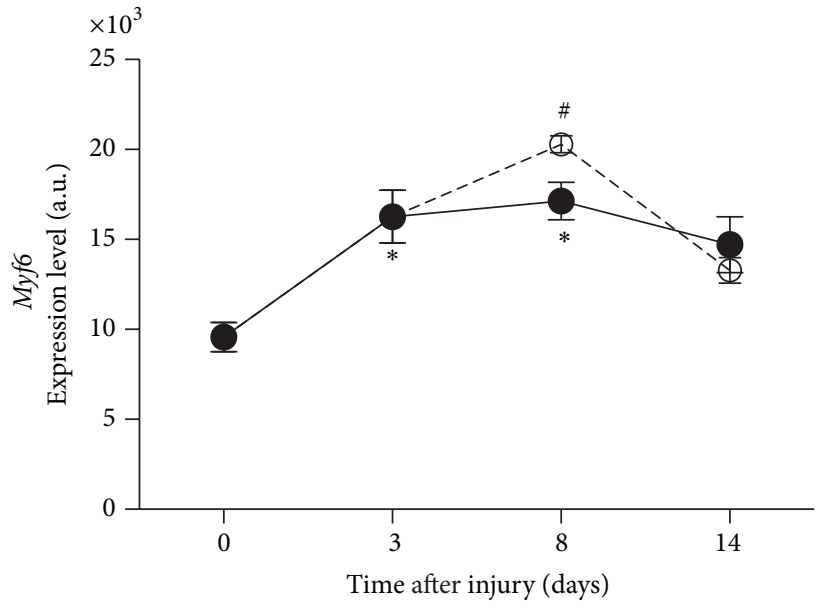

No TM

TM

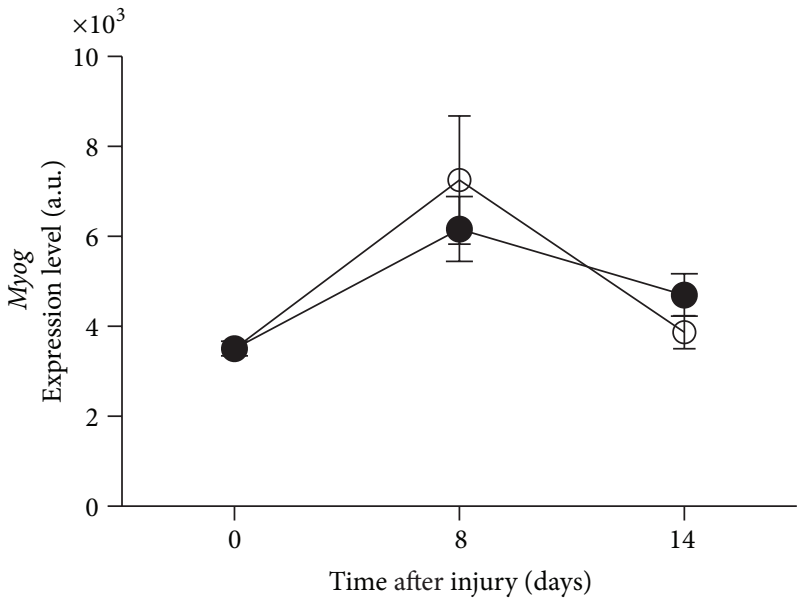

No TM

TM

(a)

(b)

FIGURE 4: Myogenic regulatory factors. Gene expression level changes in Myf6 (a), Myog (b), in soleus of trained and untrained SCI animals. All expression levels are referenced to a control sample's GAPDH levels. ${ }^{*}$ Significantly different from controls $(p<0.05) .{ }^{*}$ Significantly different from untrained animals $(p<0.05)$.

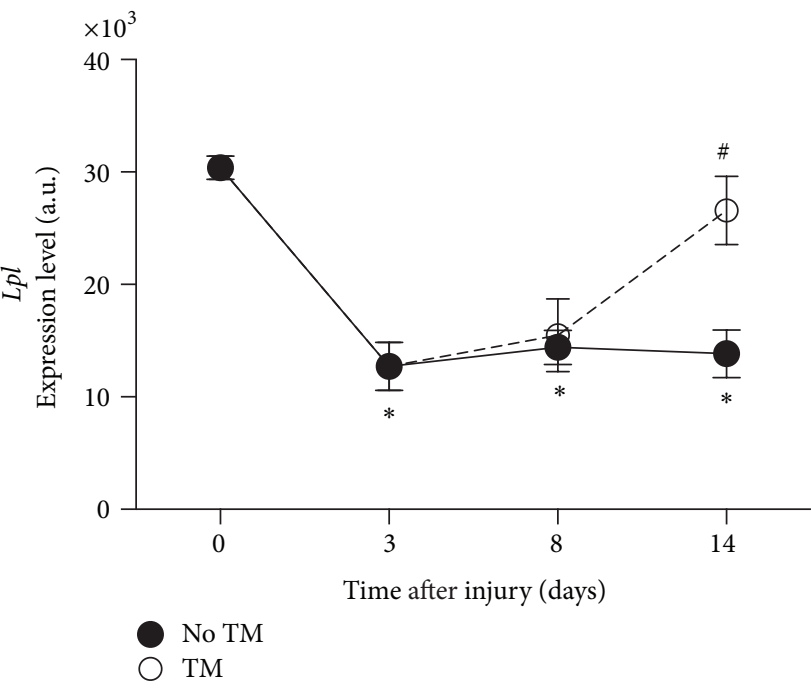

(a)

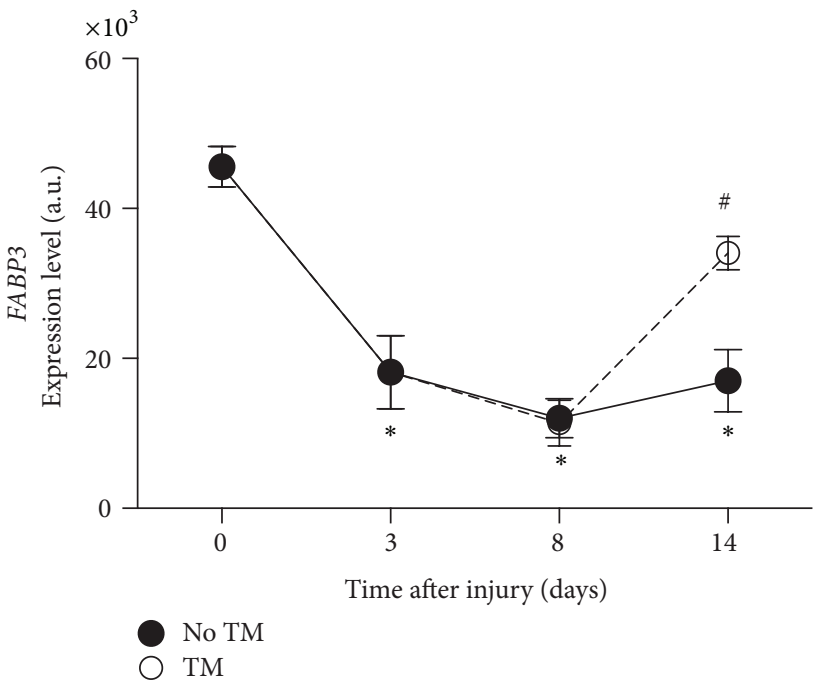

(b)

FIGURE 5: Fatty acids metabolism. Gene expression level changes in $\operatorname{Lpl}$ (a), Fabp3 (b), in soleus of trained and untrained SCI animals. All expression levels are referenced to a control sample’s GAPDH levels. ${ }^{*}$ Significantly different from controls $(p<0.05)$. ${ }^{\#}$ Significantly different from untrained animals $(p<0.05)$.

by treadmill training including Fst, Jun, Bmpr2, Actr2b, and Smad3. In addition, key players in fatty acids metabolism ( $L p l$ and Fabp3) proved to be major sensors of SCI induced inactivity and reloading with training. The decrease in Smad3 and Fst early after the initiation of treadmill training was confirmed by RT-PCR. Our data suggest that TGF- $\beta / S m a d 3$ signaling may be mainly involved in the decrease in muscle mass observed with SCI, while the BMP pathway was activated with treadmill training. We also identified changes in fiber type markers, consistent with a switch towards type II fibers with SCI, and a reverse effect of treadmill training at the gene expression level as early as 1 day after initiation.

4.1. Acute Response from the Protein Ubiquitination Pathway to SCI and Training. The protein ubiquitination pathway is essential to the control of protein breakdown and turnover in the cell. It has been established that the activation of this pathway contributes largely to muscle wasting in multiple 


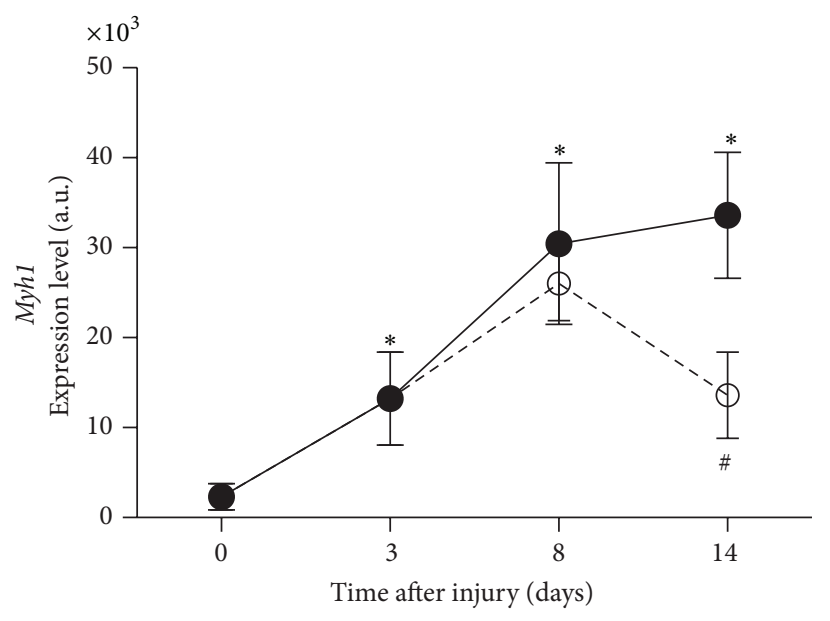

(a)

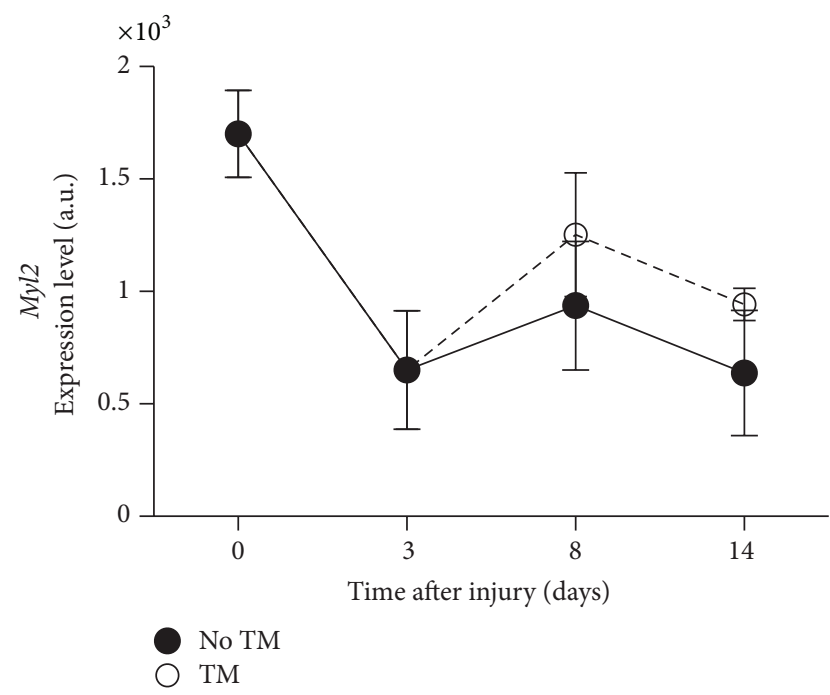

(c)

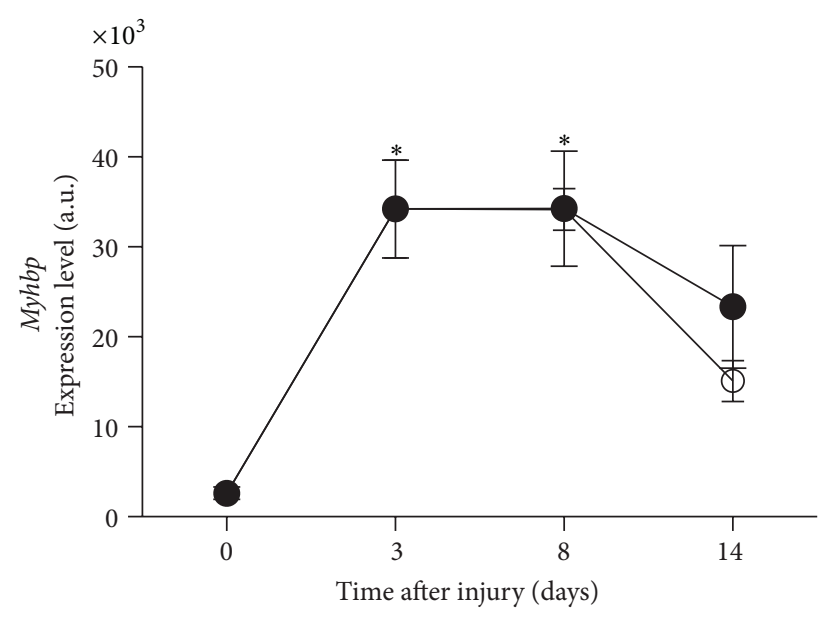

(b)

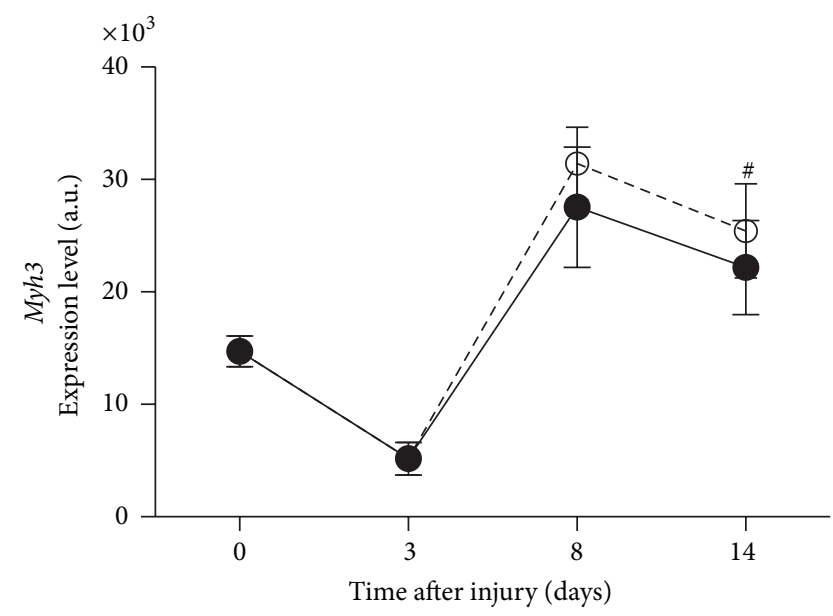

No TM

$\mathrm{TM}$

Figure 6: Myosin heavy and light chain indicative of fiber type. Gene expression level changes in Myhl (a), Myhbp (b), indicative of fast fibers, and $M y l 2$ and $M y h 3$, indicative of slow fibers in soleus of trained and untrained SCI animals. All expression levels are referenced to a control sample’s GAPDH levels. * Significantly different from controls $(p<0.05)$. "Significantly different from untrained animals $(p<0.05)$.

conditions [33], including denervation [34, 35], age and sarcopenia [36], and hindlimb suspension in rats [37] and in spinal cord injury in humans [21,38]. In our model of moderate SCI, the protein ubiquitination pathway was activated as early as 3 days after injury, with the expression of 56 genes significantly upregulated. This upregulation was most likely responsible for the unbalance in protein synthesis/degradation ratio resulting in the large muscle wasting observed in the soleus on day 3. Conversely, the expression of genes in the protein ubiquitination pathway was very rapidly decreased on day 8 after only 3 sessions of treadmill training in SCI animals and the expression of several 20 S proteasome subunits was significantly reduced. This was in accordance with previous observations in a hindlimb suspension rat model [39] 10 days after reloading. However, in another study in humans, Reich et al. [38] could not detect any reverse effect with $24 \mathrm{~h}$ of reloading after lower limb suspension.
Here, we were able to observe a significant and early response within $36 \mathrm{~h}$ after the first training session, with a significant decrease in the expression of the genes related to protein ubiquitination (Suppl. Table 5).

4.2. Normalization of Mitochondrial Function Related Pathways with Training. Genes involved in the mitochondrial dysfunction and oxidative phosphorylation related pathways were also significantly changed. Together, the large number of NUDF, SDH, and ATP synthases with changing gene expression observed in SCI demonstrates a strong metabolic activity disturbance associated with SCI that does not completely recover within 14 days after surgery. Mitochondrial dysfunction after SCI was observed in vivo in several studies performed in humans with SCI [40,41]. Our own work using ${ }^{31} \mathrm{P}$ magnetic resonance spectroscopy to assess mitochondrial function in vivo in the same rat model of moderate SCI [24] 


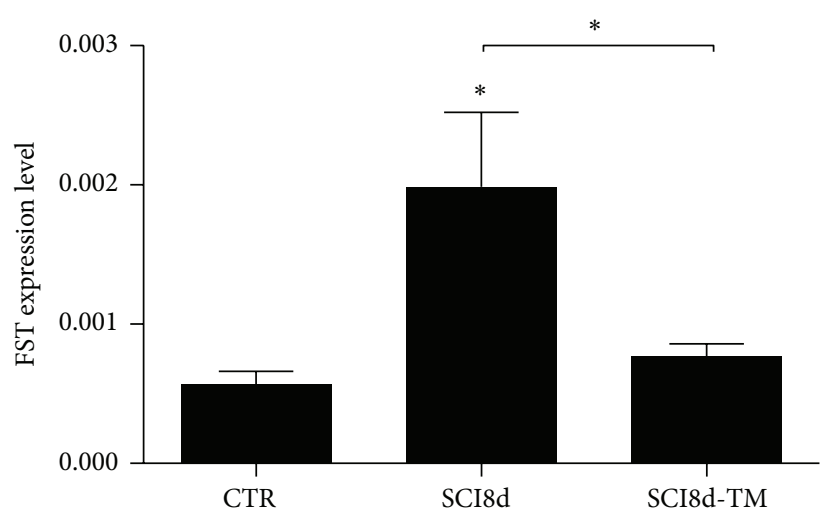

(a)

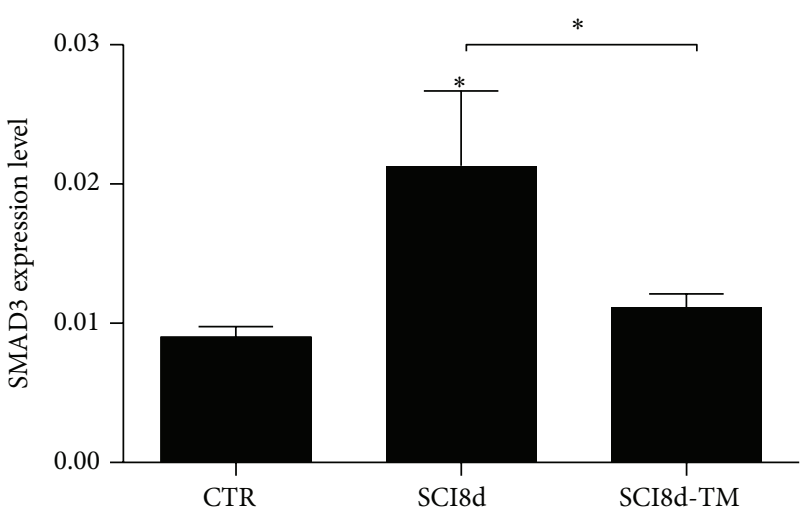

(b)

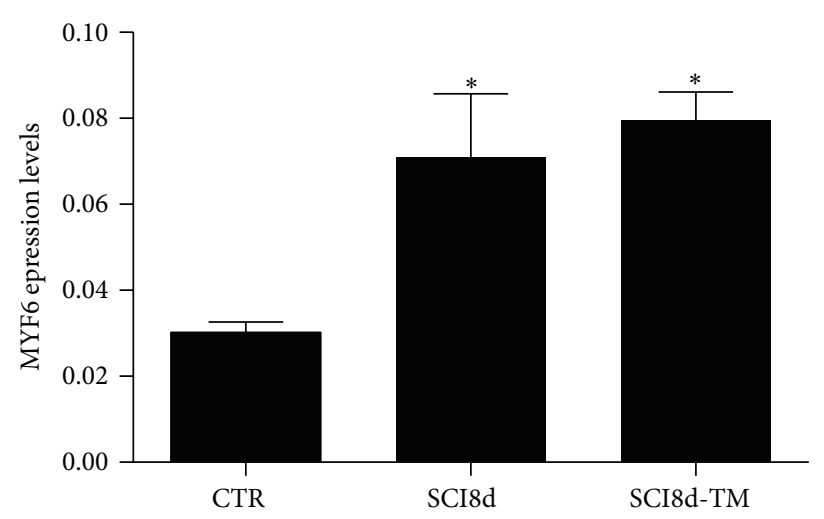

(c)

FIGURE 7: FST (a), SMAD3 (b), and MYF6 (c) mRNA expression in the soleus muscle of controls, untrained SCI animal on day 8 after surgery (SCI8d), and trained SCI animal on day 8 after surgery (SCI8d + TM). ${ }^{*} p<0.05$.

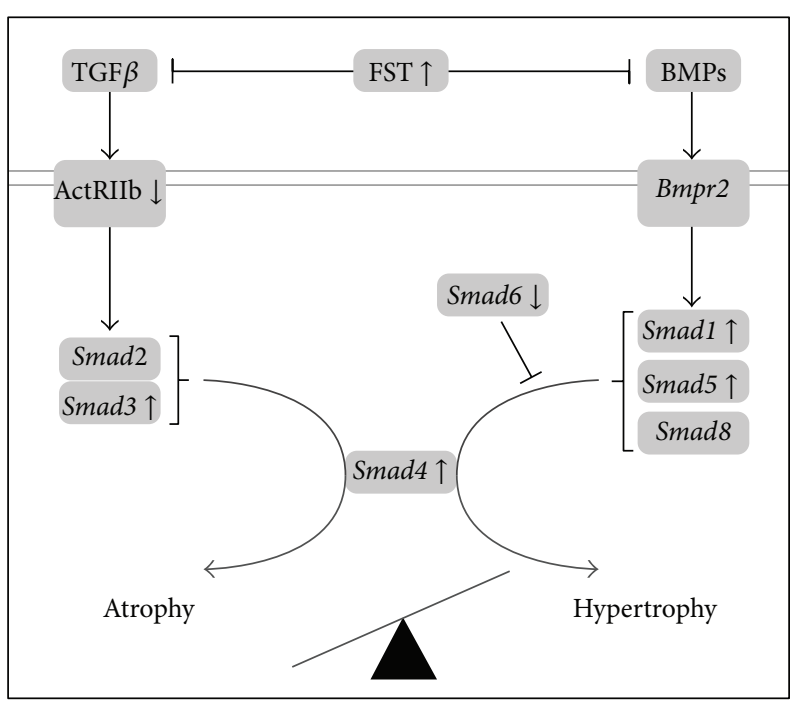

(a)

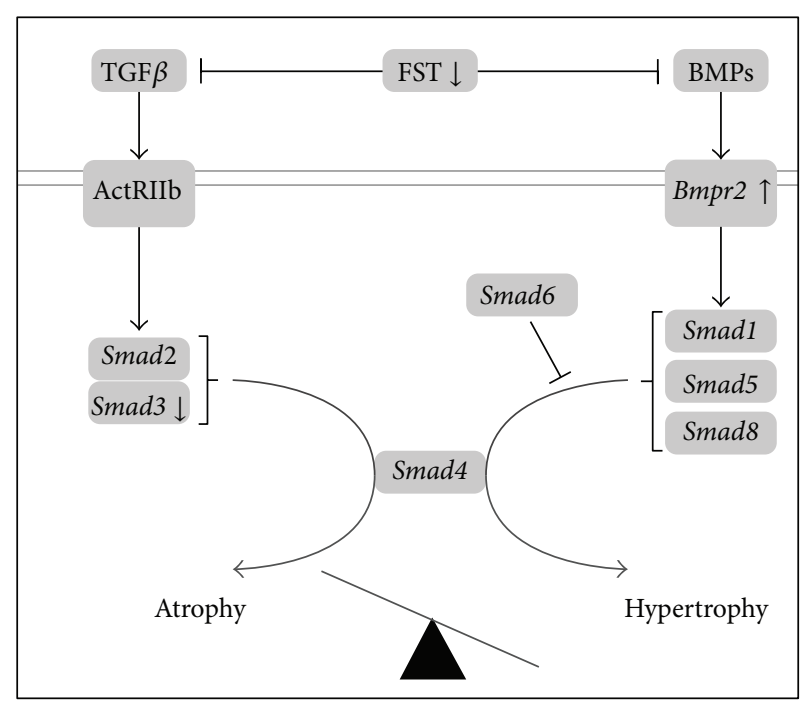

(b)

FIGURE 8: Schematic of the changes in soleus muscle gene expression within the TGF $\beta$ and BMP pathways, with SCI (a) and acute response to treadmill training (b). Panel (a) shows the combined changes observed on day 3 and 8 after SCI, where several Smads (Smads 3, 4, and 1/5) and Fst gene expression was increased compared to control in parallel with muscle atrophy. Panel (b) summarizes the changes observed 8 days after SCI after 3 sessions of treadmill training compared to untrained animals. The changes in Smad3 and Fst were reversed, and Bmpr 2 expression was increased, suggesting a role for the BMP pathway in the initiation of muscle mass recovery process observed with treadmill training. 
showed that ATP production by mitochondria was affected 1 week after SCI and recovered in 3 weeks without training. It should be noted that, in the present study, no acute effect of treadmill training on mitochondrial dysfunction and oxidative phosphorylation pathways related genes was detected on day 8 . However, our results suggest that treadmill training and the concomitant muscle hypertrophy are accompanied by partial correction of the mitochondrial function pathway genes (NUDFs and ATP synthase) at a later time (day 14), making mitochondria a target for early therapy in moderate SCI.

4.3. Role of TGF- $\beta /$ Smad and BMP Pathways in Atrophy following SCI. Follistatin (FST) is well known as an inhibitor of the myostatin signaling within the TGF $\beta$ pathway. It has been shown that Fst overexpression leads to a large increase in muscle mass in different animal models [42, 43]. Our results showed a large significant increase of FST mRNA levels with SCI after 8 days. It is possible that this increase reflects a protective mechanism intended to stimulate muscle growth. However our data showed that muscle wet weight did not further decrease after the initial 28\% loss on day 3. One possibility is that the increase in FST mRNA levels may not have been sufficient to produce a recovery of muscle mass. Interestingly, we also observed a concomitant increase in Smad3 and Smad4 gene expression, downstream the Act $2 \mathrm{~b}$ receptor, which might be responsible for atrophy. Indeed, phosphorylated SMAD3 can mediate the activation of ubiquitin ligases that induces proteasomal degradation of contractile proteins [44]. More importantly, Winbanks et al. [45] recently showed that SMAD3 protein expression prevents skeletal muscle growth induced by follistatin and may suppress Akt/mTOR/S6K signaling. In addition, Smad1 expression downstream the BMP pathway was increased, potentially competing with SMAD3 for the recruitment of SMAD4. If translated at the protein level, the balance between FST inhibition of the TGF $\beta$ signaling and increased SMAD3 and SMAD1 expression may have led to a plateau in soleus muscle mass after the initial drop (Figure 8).

4.4. Role of TGF $\beta /$ Smad and BMP Pathways in Hypertrophy following Treadmill Training. On the other hand, treadmill training was able to restore the initial muscle mass by day 14 after SCI. Our main associated findings were a large and rapid increase in the expression of Bmpr2 with treadmill training 36 hours after initiation, in parallel with the significant decrease in FST and SMAD3 mRNA levels. The increased Bmpr2 (BMPs receptor) levels could favor its activation by BMPs and the subsequent phosphorylation of Smad1, Smad5, and Smad8, which in turn bind with Smad4 leading to hypertrophy [19]. Whereas a decrease in FST expression has the ability to enable MSTN signaling and muscle growth, a parallel decrease in SMAD3 protein expression could contribute to an increase in SMAD4's availability to other binding proteins that can lead to hypertrophy, such as SMADs 1, 5, and 8. A concomitant and rapid increase in Jun was observed. It was shown that this transcription factor acts downstream the TGF $\beta$ [46] and that overexpression of JUN results in dephosphorylation of SMAD3 [47]. This body of observations is suggestive of a deactivation of the $\mathrm{TGF} \beta /$ Smad pathway and a larger role for the BMP axis in the hypertrophy process following SCI and treadmill training.

4.5. Other Growth Factors (IGFs, MRFs). The IGF1-AktmTOR pathway is known as a positive regulator of muscle mass $[18,48-50]$. This pathway was not highly ranked by the present microarray analysis. However, the group previously demonstrated the impact of moderate SCI and treadmill training on several IGF proteins and binding proteins, but not on MYF5 in skeletal muscle [23]. Consistently with these previous results, here we observed that Igbp5 and Myog were overexpressed after SCI and that Igbp5 expression levels were corrected with treadmill training. In addition, we explored the possible impact of treadmill training on MRF4/MYF6 as indicated by the microarray results and found no significant increase in mRNA levels. This discrepancy may be due to experimental variations or slight biological differences between the batches of muscle samples used for microarray and RT-qPCR validation.

4.6. Fatty Acid Metabolism and Fiber Type Related Genes as a Sensor of Muscle Activity in SCI and Treadmill Training. Lipoprotein lipase (LPL) is a major enzyme involved in fatty acid (FA) metabolism and transport. After transport into the cytoplasm, FA binds to the fatty acid binding protein 3 (FABP3). Both LPL and FABP3 have been shown to be highly sensitive to contractile activity in muscle [51, 52]. Here, both $L p l$ and Fabp3 genes showed high sensitivity to SCI induced disuse and to locomotor training. The downregulation of these fatty acid transporters in SCI rats was consistent with the recent observation by Long et al. [53] in muscle biopsies obtained from human subjects with SCI. On the other hand, we measured a large positive change in Lpl and Fabp3 gene expression in response to locomotor training, with expression levels nearly corrected (92\% of control levels) by day 14. Upregulation of LPL and FABP3 has been previously demonstrated in conjunction with increased muscle activity, such as muscle endurance training in humans [54], reloading after hindlimb suspension [28], and electrostimulation in denervated muscles [55] in rats. We established that treadmill training, as performed in our study, was sufficient to restore control levels of gene expression within 5 days. Our results further demonstrated the high sensitivity of $L p l$ and Fabp3 gene expression to muscle activity and reloading achieved by treadmill training leading to muscle hypertrophy in moderate SCI rats.

Fiber type related genes also showed a great sensitivity to SCI induced disuse. It has been well established in the literature that muscle fibers distribution undergoes a shift towards fast fibers with disuse after SCI [56]. Consistent with this, we observed a fast change in the expression of myosin heavy and light chains genes, with an increase in some of the fast type (Myhl, Myhbp) and a decrease in the slow type (Myl2 and $M y h 3)$. Interestingly, the expression level of $M y h 1$ was corrected in response to treadmill training on day 14, showing the effect of training on SCI muscles. 
In summary, using genome wide analysis, we were able to identify some of the main pathways responsible for muscle wasting following moderate SCI and more importantly the corrective effect of treadmill training on these pathways. Here, we chose to focus on the BMP and TGF $\beta$ signaling and established a key role for some of the genes within these pathways. Our observations suggest that Smad3, Bmpr2, and Fst are genes of interest in the study of moderate SCI. Protein expression and phosphorylation need to be investigated to allow further interpretation, although beyond the scope of this study. It would make it possible to test the hypotheses that Fst overexpression in SCI competes with Smad3 regulation and that this effect is reversed by treadmill training leading to muscle mass recovery. More importantly, this would elucidate whether or not treadmill training activates the BMP/Smad pathway contributing to hypertrophy. This would establish the effect of BMP signaling activation and TGF $\beta$ signaling on muscle regeneration with treadmill training in SCI via Smad 3 downregulation proving early indicators of efficient reloading in SCI rat and as a promising therapeutic approach. The body of data presented in this study constitutes a comprehensive guide to future studies targeting muscle mass preservation or recovery in moderate SCI.

\section{Disclaimer}

The content is solely the responsibility of the authors and does not necessarily represent the official views of the National Institutes of Health.

\section{Conflict of Interests}

The authors declare that there is no conflict of interests regarding the publication of this paper.

\section{Acknowledgments}

This work was funded by the Paralyzed Veterans Administration-2347, NIH/NICHD RO1HD048051, and 1R24HD050846. Yi-Wen Chen and Sachchida Nand Pandey were partially supported by NIH/NIAMS under Award number 1R01AR052027 and FSHD Society under Award number FSHS-82013-01.

\section{References}

[1] M. J. Castro, D. F. Apple Jr., E. A. Hillegass, and G. A. Dudley, "Influence of complete spinal cord injury on skeletal muscle cross-sectional area within the first 6 months of injury," European Journal of Applied Physiology and Occupational Physiology, vol. 80, no. 4, pp. 373-378, 1999.

[2] P. K. Shah, J. E. Stevens, C. M. Gregory et al., "Lower-extremity muscle cross-sectional area after incomplete spinal cord injury," Archives of Physical Medicine and Rehabilitation, vol. 87, no. 6, pp. 772-778, 2006.

[3] R. Banerjea, U. Sambamoorthi, F. Weaver, M. Maney, L. M. Pogach, and T. Findley, "Risk of stroke, heart attack, and diabetes complications among veterans with spinal cord injury,"
Archives of Physical Medicine and Rehabilitation, vol. 89, no. 8, pp. 1448-1453, 2008.

[4] C. P. Elder, D. F. Apple, C. S. Bickel, R. A. Meyer, and G. A. Dudley, "Intramuscular fat and glucose tolerance after spinal cord injury-a cross-sectional study," Spinal Cord, vol. 42, no. 12, pp. 711-716, 2004.

[5] S. Rossignol and A. Frigon, "Recovery of locomotion after spinal cord injury: some facts and mechanisms," Annual Review of Neuroscience, vol. 34, pp. 413-440, 2011.

[6] R. D. de Leon, J. A. Hodgson, R. R. Roy, and V. R. Edgerton, "Locomotor capacity attributable to step training versus spontaneous recovery after spinalization in adult cats," Journal of Neurophysiology, vol. 79, no. 3, pp. 1329-1340, 1998.

[7] K. L. Caudle, E. H. Brown, A. Shum-Siu et al., "Hindlimb immobilization in a wheelchair alters functional recovery following contusive spinal cord injury in the adult rat," Neurorehabilitation and Neural Repair, vol. 25, no. 8, pp. 729-739, 2011.

[8] K. Fouad, G. A. S. Metz, D. Merkler, V. Dietz, and M. E. Schwab, "Treadmill training in incomplete spinal cord injured rats," Behavioural Brain Research, vol. 115, no. 1, pp. 107-113, 2000.

[9] M. Liu, P. Bose, G. A. Walter, F. J. Thompson, and K. Vandenborne, "A longitudinal study of skeletal muscle following spinal cord injury and locomotor training," Spinal Cord, vol. 46, no. 7, pp. 488-493, 2008.

[10] J. Frystyk, "Exercise and the growth hormone-insulin-like growth factor axis," Medicine and Science in Sports and Exercise, vol. 42, no. 1, pp. 58-66, 2010.

[11] S. H. Lecker, V. Solomon, W. E. Mitch, and A. L. Goldberg, "Muscle protein breakdown and the critical role of the ubiquitin-proteasome pathway in normal and disease states," The Journal of Nutrition, vol. 129, no. 1, pp. 227S-237S, 1999.

[12] W. E. Mitch and A. L. Goldberg, "Mechanisms of disease: mechanisms of muscle wasting: the role of the ubiquitinproteasome pathway," The New England Journal of Medicine, vol. 335, no. 25, pp. 1897-1905, 1996.

[13] J. Batt, J. Bain, J. Goncalves et al., "Differential gene expression profiling of short and long term denervated muscle," The FASEB Journal, vol. 20, no. 1, pp. 115-117, 2006.

[14] S. H. Lecker, R. T. Jagoe, A. Gilbert et al., "Multiple types of skeletal muscle atrophy involve a common program of changes in gene expression," The FASEB Journal, vol. 18, no. 1, pp. 39-51, 2004.

[15] A. Raffaello, P. Laveder, C. Romualdi et al., "Denervation in murine fast-twitch muscle: short-term physiological changes and temporal expression profiling," Physiological Genomics, vol. 25, no. 1, pp. 60-74, 2006.

[16] H. Tang, W. M. W. Cheung, F. C. F. Ip, and N. Y. Ip, "Identification and characterization of differentially expressed genes in denervated muscle," Molecular and Cellular Neurosciences, vol. 16, no. 2, pp. 127-140, 2000.

[17] Y.-W. Chen, M. J. Hubal, E. P. Hoffman, P. D. Thompson, and P. M. Clarkson, "Molecular responses of human muscle to eccentric exercise," Journal of Applied Physiology, vol. 95, no. 6, pp. 2485-2494, 2003.

[18] S. Schiaffino, K. A. Dyar, S. Ciciliot, B. Blaauw, and M. Sandri, "Mechanisms regulating skeletal muscle growth and atrophy," The FEBS Journal, vol. 280, no. 17, pp. 4294-4314, 2013.

[19] R. Sartori, E. Schirwis, B. Blaauw et al., "BMP signaling controls muscle mass," Nature Genetics, vol. 45, no. 11, pp. 1309-1321, 2013. 
[20] P. Bialek, C. Morris, J. Parkington et al., "Distinct protein degradation profiles are induced by different disuse models of skeletal muscle atrophy," Physiological Genomics, vol. 43, no. 19, pp. 1075-1085, 2011.

[21] M. L. Urso, Y.-W. Chen, A. G. Scrimgeour, P. C. Lee, K. F. Lee, and P. M. Clarkson, "Alterations in mRNA expression and protein products following spinal cord injury in humans," The Journal of Physiology, vol. 579, no. 3, pp. 877-892, 2007.

[22] D. M. Basso, M. S. Beattie, and J. C. Bresnahan, "Graded histological and locomotor outcomes after spinal cord contusion using the NYU weight-drop device versus transection," Experimental Neurology, vol. 139, no. 2, pp. 244-256, 1996.

[23] M. Liu, J. E. Stevens-Lapsley, A. Jayaraman et al., "Impact of treadmill locomotor training on skeletal muscle IGF1 and myogenic regulatory factors in spinal cord injured rats," European Journal of Applied Physiology, vol. 109, no. 4, pp. 709-720, 2010.

[24] P. K. Shah, F. Ye, M. Liu et al., "In vivo ${ }^{31}$ P NMR spectroscopy assessment of skeletal muscle bioenergetics after spinal cord contusion in rats," European Journal of Applied Physiology, vol. 114, no. 4, pp. 847-858, 2014.

[25] D. M. Basso, M. S. Beattie, J. C. Bresnahan et al., "MASCIS evaluation of open field locomotor scores: effects of experience and teamwork on reliability. Multicenter Animal Spinal Cord Injury Study," Journal of Neurotrauma, vol. 13, no. 7, pp. 343359, 1996.

[26] M. Liu, P. Bose, G. A. Walter, D. K. Anderson, F. J. Thompson, and K. Vandenborne, "Changes in muscle T2 relaxation properties following spinal cord injury and locomotor training," European Journal of Applied Physiology, vol. 97, no. 3, pp. 355361, 2006.

[27] F. J. Thompson, P. J. Reier, C. C. Lucas, and R. Parmer, "Altered patterns of reflex excitability subsequent to contusion injury of the rat spinal cord," Journal of Neurophysiology, vol. 68, no. 5, pp. 1473-1486, 1992.

[28] L. Bey and M. T. Hamilton, "Suppression of skeletal muscle lipoprotein lipase activity during physical inactivity: a molecular reason to maintain daily low-intensity activity," The Journal of Physiology, vol. 551, no. 2, pp. 673-682, 2003.

[29] J. G. Burniston, T. H. Meek, S. N. Pandey et al., "Gene expression profiling of gastrocnemius of 'minimuscle' mice," Physiological Genomics, vol. 45, no. 6, pp. 228-236, 2013.

[30] Y.-W. Chen, K. Nagaraju, M. Bakay et al., "Early onset of inflammation and later involvement of TGF $\beta$ in Duchenne muscular dystrophy," Neurology, vol. 65, no. 6, pp. 826-834, 2005.

[31] Y.-W. Chen, P. Zhao, R. Borup, and E. P. Hoffman, "Expression profiling in the muscular dystrophies: identification of novel aspects of molecular pathophysiology," Journal of Cell Biology, vol. 151, no. 6, pp. 1321-1336, 2000.

[32] M. Dixit, E. Ansseau, A. Tassin et al., "DUX4, a candidate gene of facioscapulohumeral muscular dystrophy, encodes a transcriptional activator of PITXI," Proceedings of the National Academy of Sciences of the United States of America, vol. 104, no. 46, pp. 18157-18162, 2007.

[33] R. T. Jagoe and A. L. Goldberg, "What do we really know about the ubiquitin-proteasome pathway in muscle atrophy?" Current Opinion in Clinical Nutrition and Metabolic Care, vol. 4, no. 3, pp. 183-190, 2001.

[34] H. M. Argadine, N. J. Hellyer, C. B. Mantilla, W.-Z. Zhan, and G. C. Sieck, "The effect of denervation on protein synthesis and degradation in adult rat diaphragm muscle," Journal of Applied Physiology, vol. 107, no. 2, pp. 438-444, 2009.
[35] R. Medina, S. S. Wing, A. Haas, and A. L. Goldberg, "Activation of the ubiquitin-ATP-dependent proteolytic system in skeletal muscle during fasting and denervation atrophy," Biomedica Biochimica Acta, vol. 50, no. 4-6, pp. 347-356, 1991.

[36] M. Altun, H. C. Besche, H. S. Overkleeft et al., "Muscle wasting in aged, sarcopenic rats is associated with enhanced activity of the ubiquitin proteasome pathway," The Journal of Biological Chemistry, vol. 285, no. 51, pp. 39597-39608, 2010.

[37] D. Taillandier, E. Aurousseau, D. Meynial-Denis et al., "Coordinate activation of lysosomal, $\mathrm{Ca}^{2+}$-activated and ATPubiquitin-dependent proteinases in the unweighted rat soleus muscle," Biochemical Journal, vol. 316, no. 1, pp. 65-72, 1996.

[38] K. A. Reich, Y.-W. Chen, P. D. Thompson, E. P. Hoffman, and P. M. Clarkson, "Forty-eight hours of unloading and $24 \mathrm{~h}$ of reloading lead to changes in global gene expression patterns related to ubiquitination and oxidative stress in humans," Journal of Applied Physiology, vol. 109, no. 5, pp. 1404-1415, 2010.

[39] E. Vazeille, A. Codran, A. Claustre et al., "The ubiquitinproteasome and the mitochondria-associated apoptotic pathways are sequentially downregulated during recovery after immobilization-induced muscle atrophy," The American Journal of Physiology: Endocrinology and Metabolism, vol. 295, no. 5, pp. E1181-E1190, 2008.

[40] K. K. McCully, T. K. Mulcahy, T. E. Ryan, and Q. Zhao, "Skeletal muscle metabolism in individuals with spinal cord injury," Journal of Applied Physiology, vol. 111, no. 1, pp. 143-148, 2011.

[41] J. L. Olive, J. M. Slade, G. A. Dudley, and K. K. McCully, "Blood flow and muscle fatigue in SCI individuals during electrical stimulation," Journal of Applied Physiology, vol. 94, no. 2, pp. 701-708, 2003.

[42] A. M. Haidet, L. Rizo, C. Handy et al., "Long-term enhancement of skeletal muscle mass and strength by single gene administration of myostatin inhibitors," Proceedings of the National Academy of Sciences of the United States of America, vol. 105, no. 11, pp. 4318-4322, 2008.

[43] S.-J. Lee and A. C. McPherron, "Regulation of myostatin activity and muscle growth," Proceedings of the National Academy of Sciences of the United States of America, vol. 98, no. 16, pp. 93069311, 2001.

[44] S. Lokireddy, C. McFarlane, X. Ge et al., "Myostatin induces degradation of sarcomeric proteins through a Smad3 signaling mechanism during skeletal muscle wasting," Molecular Endocrinology, vol. 25, no. 11, pp. 1936-1949, 2011.

[45] C. E. Winbanks, J. L. Chen, H. Qian et al., "The bone morphogenetic protein axis is a positive regulator of skeletal muscle mass," The Journal of Cell Biology, vol. 203, no. 2, pp. 345-357, 2013.

[46] L. Li, J.-S. Hu, and E. N. Olson, "Different members of the jun proto-oncogene family exhibit distinct patterns of expression in response to type beta transforming growth factor," The Journal of Biological Chemistry, vol. 265, no. 3, pp. 1556-1562, 1990.

[47] A. Raffaello, G. Milan, E. Masiero et al., "JunB transcription factor maintains skeletal muscle mass and promotes hypertrophy," The Journal of Cell Biology, vol. 191, no. 1, pp. 101-113, 2010.

[48] D. J. Glass, "PI3 kinase regulation of skeletal muscle hypertrophy and atrophy," Current Topics in Microbiology and Immunology, vol. 346, no. 1, pp. 267-278, 2010.

[49] E. Latres, A. R. Amini, A. A. Amini et al., "Insulin-like growth factor-1 (IGF-1) inversely regulates atrophy-induced genes via the phosphatidylinositol 3-kinase/Akt/mammalian target of rapamycin (PI3K/Akt/mTOR) pathway," The Journal of Biological Chemistry, vol. 280, no. 4, pp. 2737-2744, 2005. 
[50] J. M. Sacheck, A. Ohtsuka, S. C. McLary, and A. L. Goldberg, "IGF-I stimulates muscle growth by suppressing protein breakdown and expression of atrophy-related ubiquitin ligases, atrogin-1 and MuRF1," American Journal of Physiology: Endocrinology and Metabolism, vol. 287, no. 4, pp. E591-E601, 2004.

[51] R. B. Simsolo, J. M. Ong, and P. A. Kern, "The regulation of adipose tissue and muscle lipoprotein lipase in runners by detraining," The Journal of Clinical Investigation, vol. 92, no. 5, pp. 2124-2130, 1993.

[52] E. Ernicka, E. Smol, J. Langfort, and M. Górecka, "Time course of changes in lipoprotein lipase activity in rat skeletal muscles during denervation-reinnervation," Journal of Applied Physiology, vol. 92, no. 2, pp. 535-540, 2002.

[53] Y. C. Long, E. Kostovski, H. Boon, N. Hjeltnes, A. Krook, and U. Widegren, "Differential expression of metabolic genes essential for glucose and lipid metabolism in skeletal muscle from spinal cord injured subjects," Journal of Applied Physiology, vol. 110, no. 5, pp. 1204-1210, 1985.

[54] B. Schmitt, M. Flück, J. Décombaz et al., "Transcriptional adaptations of lipid metabolism in tibialis anterior muscle of endurance-trained athletes," Physiological Genomics, vol. 15, pp. 148-157, 2004.

[55] E. Smol, E. Zernicka, D. Czarnowski, and J. Langfort, "Lipoprotein lipase activity in skeletal muscles of the rat: effects of denervation and tenotomy," Journal of Applied Physiology, vol. 90, no. 3, pp. 954-960, 2001.

[56] S. Ciciliot, A. C. Rossi, K. A. Dyar, B. Blaauw, and S. Schiaffino, "Muscle type and fiber type specificity in muscle wasting," International Journal of Biochemistry and Cell Biology, vol. 45, no. 10, pp. 2191-2199, 2013. 

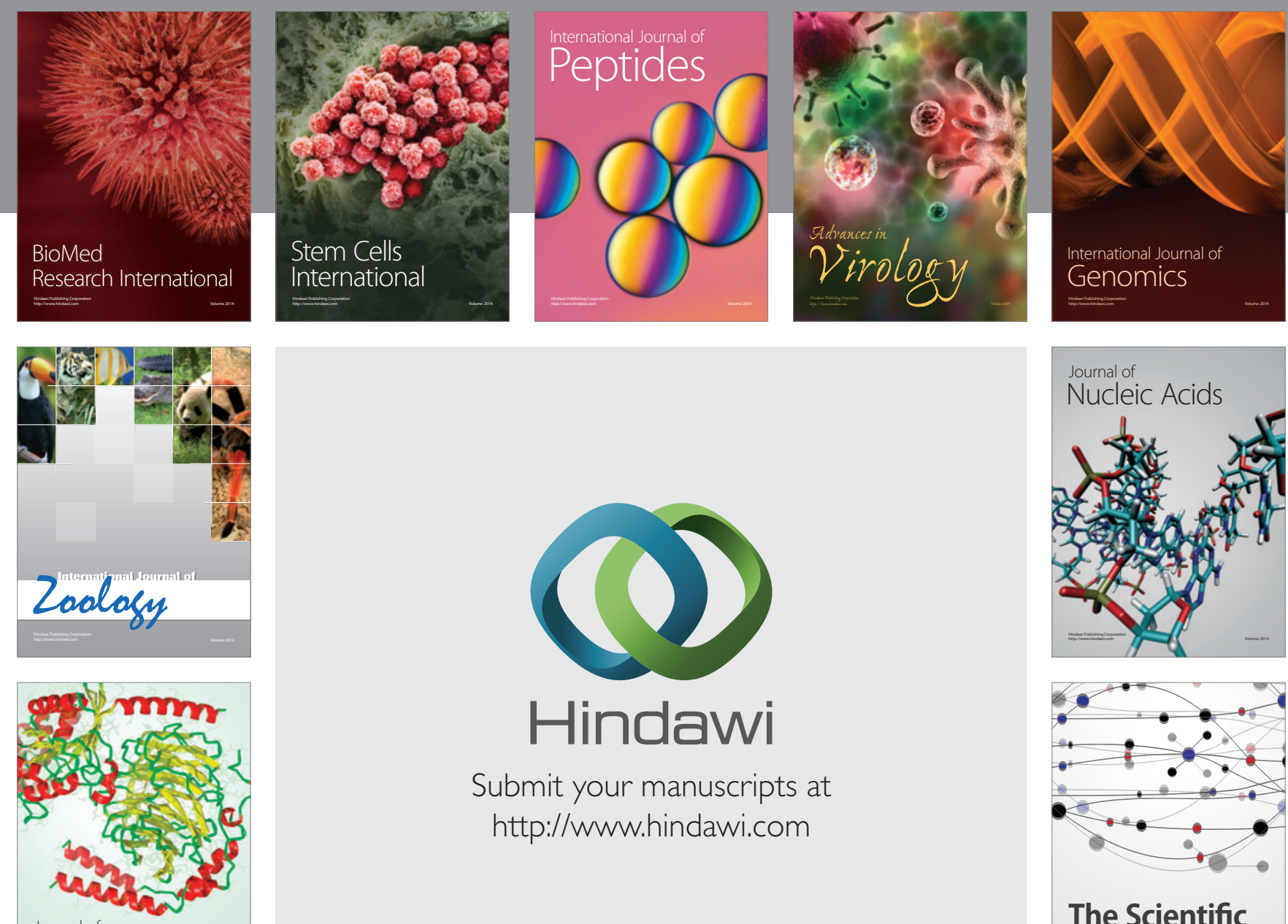

Submit your manuscripts at

http://www.hindawi.com

Journal of
Signal Transduction
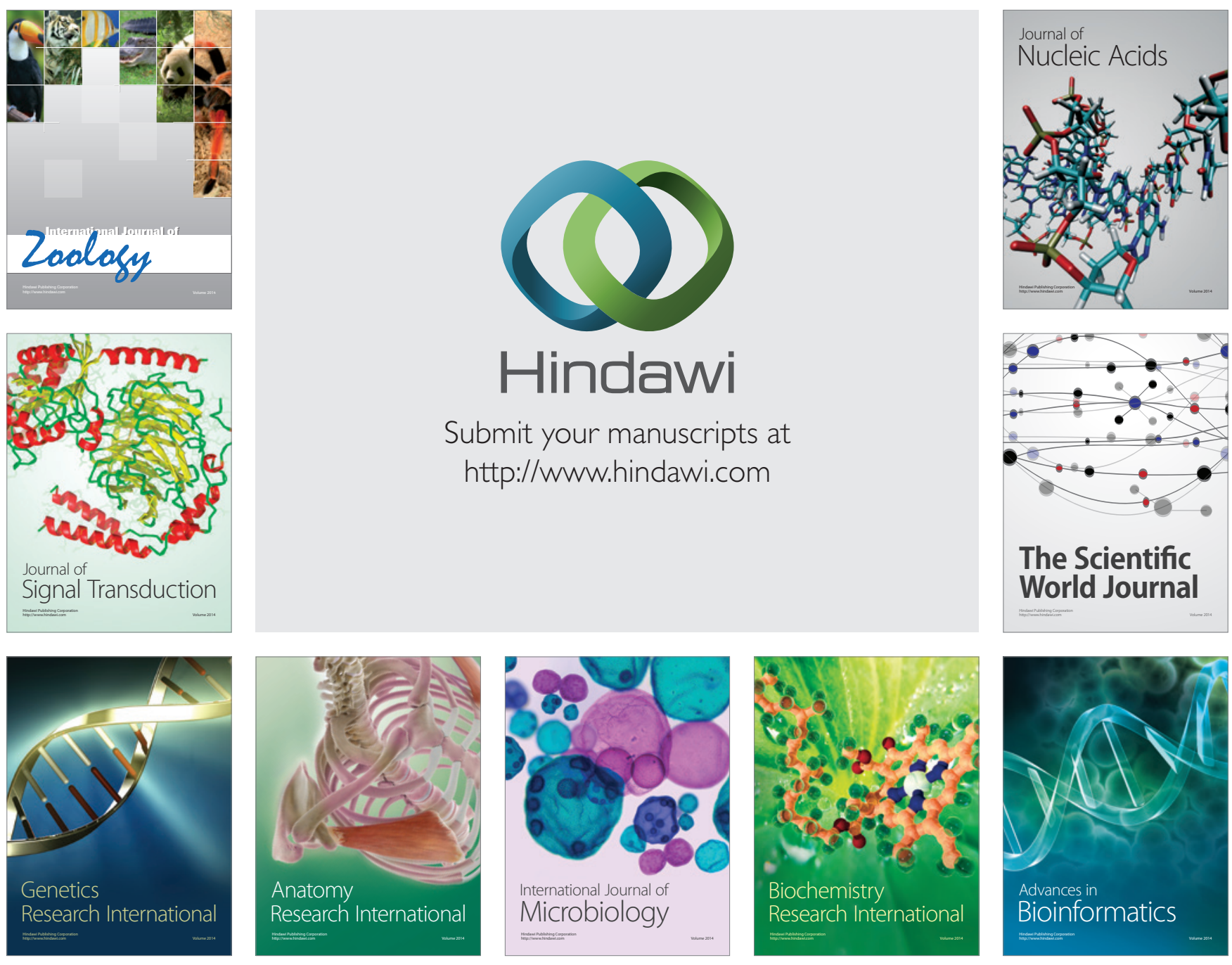

The Scientific World Journal
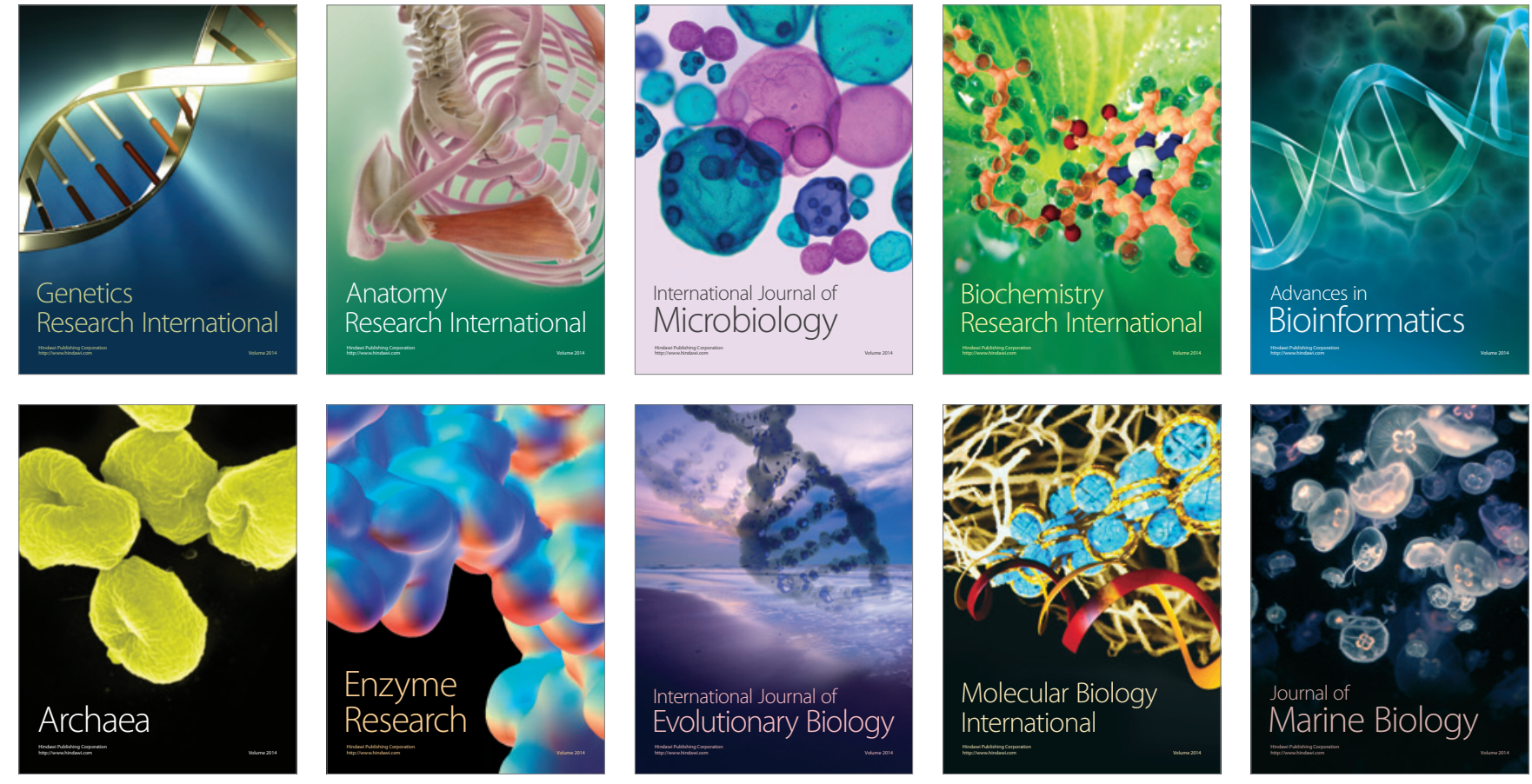